\title{
DIVERSIDAD AGRONÓMICA Y MORFOLÓGICA DE TOMATES ARRIÑONADOS Y TIPO PIMIENTO DE USO LOCAL EN PUEBLA Y OAXACA, MÉXICO
}

\author{
AGRONOMIC AND MORPHOLOGICAL DIVERSITY OF LOCAL KIDNEY \\ AND BELL PEPPER-SHAPED TOMATOES FROM PUEBLA AND OAXACA, MÉXICO
}

\author{
Olga Bonilla-Barrientos ${ }^{1}$, Ricardo Lobato-Ortiz ${ }^{1 \star}$, J. Jesús García-Zavala ${ }^{1}$, Serafín Cruz-Izquierdo ${ }^{1}$, \\ Delfino Reyes-López ${ }^{2}$, Enrique Hernández-Leal ${ }^{1}$ y Aurelio Hernández-Bautista ${ }^{1}$
}

\begin{abstract}
${ }^{1}$ Postgrado en Recursos Genéticos y Productividad-Genética, Campus Montecillo, Colegio de Postgraduados. Km. 36.5 Carr. México-Texcoco. 56230, Montecillo, Texcoco, Edo. de México. Tel. (595) 20200 Ext. 1534. ${ }^{2}$ Facultad de Ingeniería Agrohidráulica, Benemérita Universidad Autónoma de Puebla. San Juan Acateno, Teziutlán, Puebla.
\end{abstract}

${ }^{*}$ Autor para correspondencia (rlobato@colpos.mx)

\section{RESUMEN}

En algunas regiones de los Estados de Puebla y Oaxaca en México, los tomates (Solanum lycopersicum L.) nativos conocidos como "arriñonados", "chinos criollos", "cuadrados" o "tipo pimiento", "ojo de venado" y "cereza", se usan ampliamente y son cultivados para autoconsumo y comercialización local. Estos materiales pueden ser fuentes de germoplasma para usarse en programas de mejoramiento genético. Con el objetivo de describir la diversidad agronómica y morfológica de tomates nativos de amplio uso local en Puebla y Oaxaca, se evaluaron 40 colectas más el híbrido comercial 'SUN7705' como testigo, en un diseño experimental de bloques completos al azar con cuatro repeticiones y cinco plantas por repetición. Para la caracterización agronómica y morfológica de los genotipos se midieron 20 variables (diámetro de planta, altura a los $95 \mathrm{~d}$, altura al primer racimo, distancia entre racimos, días a floración del tercer racimo, número de flores del tercer racimo, días a maduración del fruto del tercer racimo, número de frutos del tercer racimo, número de racimos totales, número de hojas, número de pétalos, longitud del racimo, número total de frutos y peso total de frutos, peso promedio del fruto, diámetro, longitud y firmeza del fruto, número de lóculos y sólidos solubles totales). Mediante un análisis de varianza combinado se detectó que hubo diferencias significativas $(P \leq 0.01)$ entre genotipos, para la mayoría de las variables. Los dos primeros componentes principales explicaron $77.03 \%$ de la variación entre las colectas. Se identificaron colectas sobresalientes en sólidos solubles totales y precocidad, en materiales con frutos tipo "cereza", "ojo de venado" y "arriñonados", que podrían utilizarse directamente como variedades de uso local o como fuente de germoplasma. Los tomates con frutos "cuadrados" o "tipo pimiento" sobresalieron en tamaño del fruto, sólidos solubles y firmeza, y sus características fueron similares al testigo ('SUN7705'), por lo que tienen potencial para ser usados directamente como variedades o fuente de germoplasma para el mejoramiento genético de tomates tipo "saladette", los cuales son de amplio uso a nivel nacional.

Palabras clave: Solanum lycopersicum, diversidad, tomates nativos mexicanos.

\section{SUMMARY}

Native or landrace tomatoes (Solanum lycopersicum L.) known as "deer eye", "cherry", "kidney" and "pepper-shaped" are widely used in the States of Puebla and Oaxaca, México, where they are cultivated for self-consumption and local commerce. These materials represent an important source of germplasm for tomato breeding programs. To describe the agronomic and morphological diversity of widely used landrace tomatoes in Puebla and Oaxaca, 40 accessions plus the commercial hybrid 'SUN7705' were evaluated in a randomized complete block design with four replications and five plants per replicate. In order to characterize the morphology and agronomy of these genotypes, 20 traits were measured (plant diameter, height at $95 \mathrm{~d}$, height to the first cluster, distance between clusters, days to flowering of the third cluster, number of flowers of the third cluster, days to maturity of the third cluster, number of fruits of the third cluster, total number of clusters, number of leaves, number of petals, cluster length, total number and total weight of fruits, average fruit weight, diameter, length and firmness of the fruit, number of locules, and total soluble solids). Variance was calculated through a combined analysis. Most traits showed significant differences $(P \leq \mathbf{0 . 0 1})$ among genotypes. A principal component analysis showed that the first two components explained $77.03 \%$ of the variation among collections. Materials with outstanding features in total soluble solids and precocity were identified in some "cherry", "deer eye" and "kidney" type collections, that might be used directly as local varieties or as a source of germplasm. Bell pepper type tomatoes excelled in fruit size, soluble solids, firmness, with characteristics similar to the control hybrid ('SUN7705'), so they also have potential for being used directly as varieties or source of germplasm for genetic improvement in "saladette" tomatoes, which are widely used at the national level.

Index words: Solanum lycopersicum, diversity, Mexican native tomatoes.

\section{INTRODUCCIÓN}

El tomate (Solanum lycopersicum L.) es originario de la planicie costera occidental de América del Sur, pero se considera a México como su centro de domesticación (Jenkins, 1948; Rick y Fobes, 1975; Peralta y Spooner, 2007). La palabra jitomate se utiliza en el centro de México; en el resto del mundo hispanoparlante se usa el nombre común tomate.

Entre las hortalizas el tomate ocupa la mayor superficie sembrada a nivel mundial (FAOSTAT, 2008), y en México se siembran anualmente 80,000 ha en campo abierto con 
un rendimiento promedio de $28.7 \mathrm{t} \mathrm{ha}^{-1}$, para ser la segunda hortaliza más importante después del chile (Capsicum annuum L.), por su superficie sembrada, por su volumen y valor de producción en el mercado nacional, y por los empleos que genera (Nieto y Velasco, 2006; Hernández-Leal et al., 2013).

El tomate cultivado (comercial) ha experimentado severos cuellos de botella genéticos a través del proceso de domesticación y selección. Además, se poliniza por autogamia (Rick, 1976; Bai y Lindhout, 2007). Estos dos factores se combinaron para que el genoma del tomate sea muy pobre genéticamente (Miller y Tanksley, 1990) y tenga muy poca diversidad genética. Con técnicas de secuenciación se ha verificado que existe muy poco polimorfismo al nivel de ADN (Park et al., 2004; García-Martínez et al., 2005). Ante esta situación, los parientes silvestres de los cultivos son una de las principales fuentes de germoplasma para el mejoramiento de cultivos (Hoyt, 1992), porque han desarrollado múltiples características que les han permitido sobrevivir en condiciones extremas, así como resistir a plagas y enfermedades (Eigenbrode et al., 1993; Pérez et al., 1997).

Los parientes silvestres del tomate cultivado son nativos del oeste de América del Sur a lo largo de la costa del Pacífico y de los Andes altos del centro de Ecuador, a través de Perú hasta el norte de Chile y las islas Galápagos e incluyen a Solanum cheesmaniae (L. Riley) Fosberg, S. galapagense S.C. Darwin \& Peralta, S. pimpinellifolium L., S. arcanum Peralta, S. chilense (Dunal) Reiche, S. chmielewskii (C.M. Rick, Kesicki, Fobes \& M. Holle) D. M. Spooner, G. J. Anderson \& R. K. Jansen, S. corneliomulleri J. F. Macbr., S. habrochaites S. Knapp \& D. M Spooner, S. neorickii D. M. Spooner, G. J. Anderson \& R. K. Jansen, S. pennellii Correll, y S. peruvianum L. (Peralta y Spooner, 2007).

En México, el tomate silvestre se encuentra ampliamente distribuido tanto en zonas de vegetación natural como asociado con campos de cultivo donde se le considera como maleza (Sánchez-Peña et al., 2006). La mayoría de las poblaciones se han colectado en altitudes entre 0 a $1200 \mathrm{~m}$ (Sánchez-Peña et al., 2006). El germoplasma nativo es de importancia por la heterogeneidad biológica, económica y cultural de la agricultura local (Bellon, 1996). Constituye un recurso potencialmente valioso para la obtención de variedades mejoradas (Hoyt, 1992).

En el país se han hecho algunos estudios sobre los recursos genéticos de la especie. En uno de ellos se estudiaron siete tipos de tomate originarios de los Estados de Guerrero y Puebla, donde los contenidos de sólidos solubles variaron de 5.8 a $8.0^{\circ}$ Brix y la firmeza de 4.1 a 7.7 (Juárez-López et al., 2009), valores que son considerados altos. Por su parte, Álvarez-Hernández et al. (2009) identificaron diversas especies de plaga asociadas al tomate silvestre, tales como Bemisia tabaci, Lyriomiza sativae y Bactericera cockerelli, las cuales no obstruyeron la fructificación. Sánchez-Peña et al. (2006) encontraron una menor incidencia de mosquita blanca (Bemisia tabaci) en tomate silvestre tipo "cherry" de Sinaloa que en el tomate cultivado variedad 'Río Grande’.

El objetivo del presente trabajo fue estudiar la diversidad agronómica y morfológica de materiales de tomate nativo de amplio uso local en los Estados de Puebla y Oaxaca, con miras a su aprovechamiento en programas nacionales de mejoramiento en el corto, mediano y largo plazo.

\section{MATERIALES Y MÉTODOS}

\section{Material vegetal}

Se trabajó con 40 colectas de tomate nativo, de las cuales 18 fueron de tomates "cuadrados" o tipo "pimiento", 15 fueron de tipo "riñón", 5 de "ojo de venado", y 2 de "cherry" o "cereza". Del total de colectas, 35 fueron de Puebla y cinco de Oaxaca. Además, se incluyó como testigo al híbrido comercial 'SUN7705' tipo "saladette" de hábito de crecimiento indeterminado (Cuadro 1). Este germoplasma está disponible en los Centros de Conservación de Semillas Ortodoxas Región Occidente (CUCBA-UDG) y Región Centro (BANGEV-UACh) del Sistema Nacional de Recursos Fitogenéticos (SINAREFI).

\section{Ubicación}

El experimento se lleva cabo durante los periodos Primavera-Verano 2011 y Otoño-Invierno 2011, en invernaderos ubicados en Montecillo, Texcoco, Estado de México, cuya localización geográfica es $19^{\circ} 30^{\prime} \mathrm{N}$ y $98^{\circ} 53^{\prime} \mathrm{O}$, a una altitud de $2250 \mathrm{~m}$.

\section{Diseño experimental y manejo agronómico}

El experimento se estableció en un diseño de bloques completos al azar con 41 tratamientos, cuatro repeticiones y cinco plantas por repetición. Se sembró en condiciones de hidroponía, en bolsas de polietileno de color negro de $12 \mathrm{~L}$ rellenas con tezontle rojo fino como sustrato. Se establecieron dos ciclos de cultivo. El primero se trasplantó el 19 de abril de 2011, y el segundo el 9 de septiembre del mismo año. En ambos ciclos, el trasplante se hizo a los $35 \mathrm{~d}$ después de la siembra. Se utilizó la solución nutritiva propuesta por Steiner (1984) para ambos ciclos. En la etapa vegetativa, se usó una concentración de $50 \%$, y en las etapas de floración y fructificación se incrementó a 100 \%.

El pH de la solución se mantuvo en un intervalo de 5.5 a 6.0. Las plantas fueron tutoradas con el objetivo 
Cuadro 1. Colectas de tomate proveniente de los Estados de Puebla y Oaxaca, utilizadas para la caracterización agronómica.

\begin{tabular}{|c|c|c|c|}
\hline Identificación & Localidad & Municipio & Nombre local \\
\hline LOR-77 & La Ceiba & Xicotepec, Pue. & Riñón \\
\hline LOR-78 & La Ceiba & Xicotepec, Pue. & O. V. \\
\hline LOR-79 & Zinacatepec & Zinacatepec, Pue. & C. C. \\
\hline LOR-80 & Altepexi & Altepexi, Pue. & C. C. \\
\hline LOR-81 & Altepexi & Altepexi, Pue. & C. C. \\
\hline LOR-82 & Altepexi & Altepexi, Pue. & Riñón \\
\hline LOR-83 & Teotitlán de Flores Magón & Teotitlán de Flores Magón, Oax. & O. V. \\
\hline LOR-84 & Altepexi & Altepexi, Pue. & C. C. \\
\hline LOR-85 & Altepexi & Altepexi, Pue. & C. C. \\
\hline LOR-86 & Zinacatepec & Zinacatepec, Pue. & O. V. \\
\hline LOR-87 & Altepexi & Altepexi, Pue. & C. C. \\
\hline LOR-88 & Teotitlán de Flores Magón & Teotitlán de Flores Magón, Oax. & Cherry \\
\hline LOR-90 & Altepexi & Altepexi, Pue. & Riñón \\
\hline LOR-91 & Altepexi & Altepexi, Pue. & C. C. \\
\hline LOR-92 & Teotitlán de Flores Magón & Teotitlán de Flores Magón, Oax. & Cherry \\
\hline LOR-95 & Tehuacán & Tehuacán, Pue. & C. C. \\
\hline LOR-97 & Santa Cruz Xitla & Santa Cruz Xitla, Oax. & Riñón \\
\hline LOR-98 & Santa Cruz Xitla & Santa Cruz Xitla, Oax. & Riñón \\
\hline LOR-99 & Santa Cruz Xitla & Santa Cruz Xitla, Oax. & Riñón \\
\hline LOR-100 & Santa Cruz Xitla & Santa Cruz Xitla, Oax. & Riñón \\
\hline LOR-101 & Miahuatlán de Porfirio Díaz & Miahuatlán de Porfirio Díaz, Oax. & Riñón \\
\hline LOR-102 & Sta. María Coapan & Tehuacán, Pue. & C. C. \\
\hline LOR-103 & Sta. María Coapan & Tehuacán, Pue. & C. C. \\
\hline LOR-104 & San Gabriel Chilac & San Gabriel Chilac, Pue. & C. C. \\
\hline LOR-106 & Altepexi & Altepexi, Pue. & C. C. \\
\hline LOR-107 & Altepexi & Altepexi, Pue. & C. C. \\
\hline LOR-108 & San Diego Chalma & Tehuacán, Pue. & C. C. \\
\hline LOR-109 & San Diego Chalma & Tehuacán, Pue. & C. C. \\
\hline LOR-110 & Zinacatepec & Zinacatepec, Pue. & C. C. \\
\hline LOR-111 & Zinacatepec & Zinacatepec, Pue. & C. C. \\
\hline LOR-113 & Altepexi & Altepexi, Pue. & C. C. \\
\hline LOR-116 & Necaxa & Juan Galindo, Pue. & Riñón \\
\hline LOR-117 & Necaxa & Juan Galindo, Pue. & Riñón \\
\hline LOR-118 & La Ceiba & Xicotepec, Pue. & O. V. \\
\hline LOR-119 & La Ceiba & Xicotepec, Pue. & Riñón \\
\hline LOR-120 & Huauchinango & Huauchinango, Pue. & Riñón \\
\hline LOR-121 & Huauchinango & Huauchinango, Pue. & Riñón \\
\hline LOR-122 & Huauchinango & Huauchinango, Pue. & O. V \\
\hline LOR-123 & Huauchinango & Huauchinango, Pue. & Riñón \\
\hline LOR-124 & Necaxa & Juan Galindo, Pue. & Riñón \\
\hline Híbrido & SUN 7705 & & Saladette \\
\hline
\end{tabular}


de dirigirlas en un solo eje. Se aplicaron fungicidas y plaguicidas como Captan $\AA$, Confidor ${ }^{\circledR}$, Beleaf $\AA$, Cupravit $\AA$, Ampligo ${ }^{\circledR}$, Previcur ${ }^{\circledR}$ en forma preventiva, para el control de mosca blanca (Bemisia tabaci Gennadius) (Hemiptera, Aleyrodidae), paratrioza (Bactericera cockerelli) y tizón tardío (Phytophthora infestans).

\section{Variables evaluadas}

Se evaluaron 20 características, de acuerdo con el manual de descriptores del tomate (Solanum lycopersicum) del International Plant Genetic Resources Institute (IPGRI, 1996). Éstas fueron: diámetro de planta antes de la cosecha, altura de planta a los $95 \mathrm{~d}$, altura al primer racimo, distancia entre racimos, días a floración del tercer racimo, número de flores del tercer racimo, días a maduración del fruto del tercer racimo, número de frutos del tercer racimo, número de racimos totales tanto de flor como de fruto hasta el momento de la cosecha, número total de hojas, número de pétalos, longitud de racimo, número total de frutos, peso total de frutos, y peso promedio del fruto; además se midió diámetro y longitud del fruto, ambas variables obtenidas de un promedio de cinco frutos. La firmeza del fruto fue medida en newtons con un texturómetro universal marca FORCEFIVE® con un puntal cónico de $0.8 \mathrm{~mm}$, modelo FDV-30 (Greenwich, EE. UU.). También se documentó el número de lóculos y los sólidos solubles totales; este último se midió con un refractómetro digital marca PAL-1 ${ }^{\circledR}$ (Tokio, Japón) de rango 0.0 a $53.0^{\circ} \mathrm{Brix}$.

\section{Análisis estadístico}

Se analizó la varianza combinada de los datos de ambos ciclos y una comparación de medias con la prueba de Tukey $(\mathrm{P} \leq 0.05)$ con el paquete estadístico SAS v.9.0 (2002). Además, se hizo un análisis de componentes principales (CP) con las 20 variables, al usar la matriz de correlaciones mediante el procedimiento PRINCOMP de SAS. La representación gráfica de las colectas con los componentes principales uno (CP1) y dos (CP2) se hizo para apreciar las similitudes y diferencias entre las 40 colectas. Además, se hizo un análisis de conglomerados mediante la distancia euclidiana y el método de agrupamiento de UPGMA, con el paquete estadístico NTSYS ${ }^{\circledR}$ (Rohlf, 2000), con el propósito de agrupar las colectas con base en distancias euclidianas.

\section{RESULTADOS Y DISCUSIÓN}

Con los análisis de varianza se detectaron diferencias significativas $(P \leq 0.01)$ entre ciclos y colectas, para la mayoría de variables (Cuadro 2). Los valores más altos de los coeficientes de variación resultaron en sólidos solubles totales con $21.51 \%$, en firmeza con $24.38 \%$, y en número de lóculos con $39.69 \%$. Estos resultados indican que estas características tuvieron una desviación estándar mayor a 20 $\%$ de la media poblacional. El resto de las variables resultaron con un coeficiente de variación menor a $20 \%$, lo cual es un indicador de la confiabilidad de los datos experimentales, porque de acuerdo con este parámetro la desviación estándar en las poblaciones resultó menor de $20 \%$ con respecto a la media poblacional. Los datos también muestran que hay una variabilidad considerable entre materiales, la cual puede ser aprovechada en el mejoramiento del tomate, como propusieron Lobato-Ortiz et al. (2012) y CarrilloRodríguez y Chávez-Servia (2010).

Los dos primeros componentes principales explicaron $77.03 \%$ de la variación total, con $54.84 \%$ y $22.19 \%$, respectivamente (Cuadro 3). Las variables incluidas en el CP1 fueron diámetro de planta, altura al primer racimo, días a floración, días a maduración del tercer racimo, número de frutos del tercer racimo, número de racimos totales, número de hojas, número total de frutos, peso promedio de fruto, y diámetro, longitud y firmeza del fruto. El CP2 estuvo compuesto por las variables número de flores del tercer racimo (NFIR3), número de pétalos, longitud del racimo, y número de lóculos.

\section{Distribución de la diversidad}

En la representación gráfica de los dos primeros componentes principales (Figura 1), se muestra la distribución de las 40 colectas y el testigo, donde se identifican cuatro grupos. El Grupo I quedó formado por las colectas LOR77, LOR97, LOR98, LOR99, LOR100, LOR101, LOR117, LOR119, LOR121, LOR123, y LOR124. Estas colectas se caracterizaron por tener la forma arriñonada del fruto y valores máximos en variables como: número de flores por racimo, con 12.69; longitud del racimo, con $24.17 \mathrm{~cm}$; número de pétalos, con 7.65; y número de lóculos, con 4.89 . El peso promedio del fruto fue de $33.32 \mathrm{~g}$; el diámetro y la longitud de fruto fueron de $37.44 \mathrm{~mm}$ y $29.36 \mathrm{~mm}$, respectivamente. La variable de firmeza mostró valores bajos, con $0.92 \mathrm{~N}$ en promedio, inferior al promedio de los Grupos III y IV. Este mismo grupo mostró valores similares en las variables días a floración y madurez, y número de racimos totales, con respecto al testigo, lo que significa que estos materiales tuvieron atributos similares al híbrido. Por ello podrían ser utilizados para el mejoramiento genético como fuente de germoplasma o para la obtención de variedades mejoradas (Cuadro 4).

El Grupo II fue conformado por las colectas LOR78, LOR83, LOR86, LOR88, LOR92, LOR116, LOR118, LOR120, y LOR122, que correspondieron a los genotipos de frutos más pequeños. La mayoría fue "ojo de venado", con excepción de dos colectas que fueron "cherry" y de dos 


\begin{tabular}{|c|c|c|c|c|c|c|c|c|c|}
\hline FV & CICLO & COL & & CICLO x COL & ERROR & CV (\%) & Intervalo & Media & $\mathrm{DE}$ \\
\hline DP & $169.07^{\star *}$ & 10.08 & $* *$ & $1.56^{* *}$ & 0.89 & 6.72 & $11.39-15.49$ & 13.91 & 3.83 \\
\hline A95 & $7714.12^{\star *}$ & 457.56 & $* *$ & $59.19 * *$ & 10.21 & 6.76 & $128.74-204.32$ & 158.78 & 13.45 \\
\hline AR1 & $21679.62^{\star *}$ & 2475.45 & ** & $97.53 * *$ & 52.51 & 5.83 & $13.96-45.41$ & 35.65 & 5.74 \\
\hline DR & $157649.10^{\star *}$ & 4222.62 & $* *$ & $202.97 *$ & 121.40 & 6.92 & $8.41-18.39$ & 14.95 & 3.96 \\
\hline DFlR3 & $2355.70^{\star *}$ & 581.56 & ** & $54.95 * *$ & 12.02 & 9.72 & $82.68-113.55$ & 100.07 & 10.92 \\
\hline NFlR3 & $197.13^{\star *}$ & 35.29 & $* *$ & $11.46^{* *}$ & 1.28 & 7.55 & $7.06-15.54$ & 9.41 & 3.08 \\
\hline DMR3 & $117702.89^{\star *}$ & 859.33 & $* *$ & $98.06^{\star *}$ & 30.78 & 5.54 & $126.94-163.96$ & 151.39 & 12.95 \\
\hline NFrR3 & $1.38 \mathrm{~ns}$ & 36.83 & ** & $3.87^{\star *}$ & 1.31 & 12.17 & $4.95-8.62$ & 6.69 & 2.79 \\
\hline NRT & $85217.96^{* *}$ & 771.65 & $* *$ & $59.51 * *$ & 16.59 & 2.69 & $4.79-10.05$ & 6.86 & 2.65 \\
\hline $\mathrm{NH}$ & $345.60^{* *}$ & 5.33 & ** & $4.41^{\star *}$ & 0.53 & 10.81 & $30.65-39.38$ & 34.25 & 5.65 \\
\hline NP & $7.13^{\star *}$ & 23.25 & ** & $0.63 * *$ & 0.31 & 8.09 & $5.00-8.50$ & 6.41 & 2.54 \\
\hline LR & $1728.38^{* *}$ & 67.68 & ** & $6.11^{* *}$ & 2.80 & 4.88 & $13.73-26.00$ & 19.87 & 4.50 \\
\hline NTF & $1272.24^{* *}$ & 37.95 & ** & 10.33 ns & 7.28 & 7.95 & $8.87-31.76$ & 14.04 & 3.97 \\
\hline PT & $1263.92^{\star *}$ & 22.36 & $* *$ & $10.65 * *$ & 5.55 & 7.99 & $81.68-1548$ & 352.31 & 20.60 \\
\hline PPF & 0.03 & 5.23 & ** & $0.02 \mathrm{~ns}$ & 0.19 & 7.04 & $4.46-103.99$ & 53.15 & 7.30 \\
\hline DF & $0.15 \mathrm{~ns}$ & 5.81 & ** & 0.02 ns & 0.19 & 6.89 & $16.70-57.72$ & 40.79 & 6.24 \\
\hline LF & $50.64^{\star *}$ & 75.20 & ** & $15.72 * *$ & 15.72 & 12.87 & $16.70-68.73$ & 41.26 & 6.34 \\
\hline $\mathrm{F}$ & $909.39^{\star *}$ & 187.64 & $* *$ & $42.71^{* *}$ & 11.85 & 24.38 & $0.43-1.78$ & 1.15 & 1.13 \\
\hline $\mathrm{L}$ & $1692622.03^{* *}$ & 440285.04 & ** & 35877.35 ** & 20027.34 & 39.69 & $2.08-5.62$ & 3.39 & 1.87 \\
\hline B & $0.86 \mathrm{~ns}$ & 8517.44 & ** & $510.31 * *$ & 130.88 & 21.51 & $3.49-4.78$ & 4.08 & 1.90 \\
\hline GL & 1 & 40 & & 40 & 240 & 327 & & & \\
\hline
\end{tabular}

$\overline{\mathrm{FV}}=$ fuente de variación; $\mathrm{GL}=$ grados de libertad; $\mathrm{CICLO}=$ ciclos de evaluación; $\mathrm{COL}=$ colecta; $\mathrm{CICLO} \mathrm{X} \mathrm{COL}=$ interacción ciclos por colecta; ${ }^{* *} \mathrm{P} \leq 0.01$, ${ }^{\star} \mathrm{P} \leq 0.05, \mathrm{~ns}=$ no significativo; $\mathrm{DP}=$ diámetro de planta $(\mathrm{mm}) ; \mathrm{A} 95=$ altura de planta a los $95 \mathrm{~d}(\mathrm{~cm}) ; \mathrm{AR} 1=$ altura al primer racimo $(\mathrm{cm}) ; \mathrm{DR}=$ distancia entre racimos (cm); DFlR3 = días a floración del 3er racimo; NFlR3 = número de flores del 3er racimo; DMR3 = días a maduración del fruto del 3er racimo; $\mathrm{NFrR3}$ = número de frutos del 3er racimo; $\mathrm{NRT}$ = número de racimos totales (flor + fruto); $\mathrm{NH}$ = número de hojas; $\mathrm{NP}=$ número de pétalos; $\mathrm{LR}=$ longitud de racimo $(\mathrm{cm}) ; \mathrm{NTF}=$ número total de frutos; $\mathrm{PT}=$ peso total de frutos $(\mathrm{g}) ; \mathrm{PPF}=$ peso promedio de fruto $(\mathrm{g}) ; \mathrm{DF}=$ diámetro de fruto $(\mathrm{mm}) ; \mathrm{LF}=$ longitud de fruto $(\mathrm{mm}) ; \mathrm{F}=$ firmeza $(\mathrm{N}) ; \mathrm{L}=$ número de lóculos; $\mathrm{B}=$ sólidos solubles totales ( $\left.{ }^{\circ} \mathrm{Brix}\right) ; \mathrm{CV}=$ coeficiente de variación; $\mathrm{DE}=$ desviación estándar.

colectas con forma de "riñón". Estos materiales resultaron más precoces en comparación con los otros grupos, con $87.62 \mathrm{~d}$ a la floración y $139.67 \mathrm{~d}$ a maduración del fruto del tercer racimo, en contraste con el grupo más tardío que tuvo 109.75 y $160.14 \mathrm{~d}$ a floración y madurez, respectivamente. En cuanto al número de flores por racimo, este grupo fue el segundo más alto con 8.62 flores en el tercer racimo, aunque no tuvo diferencia significativa con el Grupo III y IV. Los frutos tuvieron un peso promedio de $12.32 \mathrm{~g}$; el diámetro fue de $23.44 \mathrm{~mm}$, y la longitud de $21.29 \mathrm{~mm}$, características que confieren la forma redonda de estos frutos.

Álvarez-Hernández et al. (2009) encontraron valores similares en tomate silvestre (var. cerasiforme) de Michoacán, con 2.1 a $2.4 \mathrm{~cm}$ para los frutos más grandes y $1.1 \mathrm{~cm}$ en frutos pequeños, mientras que Carrillo-Rodríguez et al. (2012) encontraron que los frutos tipo "cherry" variaron de 1.3 a $2.7 \mathrm{~cm}$ de diámetro. Por su parte, Rick et al. (1990) encontraron diámetros que variaron de 1 a $1.25 \mathrm{~cm}$, que indican redondez en los frutos.

Las variables altura al primer racimo y firmeza mostraron valores más bajos con respecto a los Grupos III y IV, con $25.39 \mathrm{~cm}$ y $0.69 \mathrm{~N}$, respectivamente. A pesar del tallo delgado, sobresalieron en altura de la planta y fueron las más precoces. El contenido promedio de sólidos solubles totales fue de $4.44^{\circ}$ Brix, que superó al testigo que tuvo 3.99. Juárez-López et al. (2009) encontraron valores en un intervalo de 5.8 a 8.0 al evaluar colectas de tipo "cherry", mientras que Crisanto-Juárez et al. (2010) encontraron valores de entre 3.8 y $8.9^{\circ}$ Brix. Esto indica que es posible hacer selección de individuos para aumentar el contenido de grados Brix.

El Grupo III estuvo formado por las colectas LOR79, LOR80, LOR81, LOR82, LOR84, LOR85, LOR87, LOR90, 


\begin{tabular}{|c|c|c|}
\hline Variables & $\mathrm{CP} 1$ & $\mathrm{CP} 2$ \\
\hline Diámetro de planta (mm) & 0.243389 & 0.09971 \\
\hline Altura de planta a los $95 \mathrm{~d}(\mathrm{~cm})$ & -0.196579 & 0.21342 \\
\hline Altura al primer racimo $(\mathrm{cm})$ & 0.276353 & -0.01666 \\
\hline Distancia entre racimos $(\mathrm{cm})$ & 0.205438 & 0.2687 \\
\hline Días a floración del 3er racimo & 0.286735 & -0.02673 \\
\hline Número de flores del 3er racimo & -0.094374 & 0.40448 \\
\hline Días a maduración del fruto del 3er racimo & 0.284805 & 0.00648 \\
\hline Número de frutos del 3er racimo & -0.071834 & 0.06098 \\
\hline Número de racimos totales (flor + fruto) & -0.293296 & 0.01476 \\
\hline Número de hojas & -0.285608 & 0.07318 \\
\hline Número de pétalos & 0.027615 & 0.44947 \\
\hline Longitud de racimo $(\mathrm{cm})$ & -0.008689 & 0.44109 \\
\hline Número total de frutos & -0.227274 & -0.06655 \\
\hline Peso total de frutos $(\mathrm{g})$ & 0.160749 & 0.02238 \\
\hline Peso promedio de fruto $(\mathrm{g})$ & 0.289779 & -0.03176 \\
\hline Diámetro de frutos (mm) & 0.292683 & 0.07417 \\
\hline Longitud de frutos (mm) & 0.290235 & -0.06677 \\
\hline Firmeza $(\mathrm{N})$ & 0.278723 & -0.02021 \\
\hline Número de lóculos & -0.053262 & 0.43783 \\
\hline Sólidos solubles totales ( $\left.{ }^{\circ} \mathrm{Brix}\right)$ & -0.1607 & -0.30626 \\
\hline Valores propios & 10.97 & 4.44 \\
\hline Varianza explicada (\%) & 54.84 & 22.19 \\
\hline Varianza acumulada (\%) & 54.84 & 77.03 \\
\hline
\end{tabular}

LOR91, LOR95, LOR102, LOR103, LOR104, LOR106, LOR107, LOR108, LOR109, LOR110, LOR111 y LOR113. La mayoría de las colectas tienen frutos tipo "cuadrado" o "pimiento", y dos colectas son con fruto "arriñonado". Estas colectas son de fruto grande con peso promedio del fruto de $81.25 \mathrm{~g}$, pero muy tardías con $109.75 \mathrm{~d}$ a floración y 160.14 d a maduración del fruto. Diámetro de planta, altura al primer racimo, número de flores del tercer racimo, longitud del racimo y contenido de sólidos solubles totales, fueron las variables con valores superiores o iguales al testigo.

Young et al. (1993) y Martínez (2003) señalaron que varios genotipos nativos de tomate producen frutos con una concentración de sólidos solubles mayor a la de las variedades cultivadas. En esta variable varios de nuestros materiales sobresalieron, posiblemente debido a la selección que por varias generaciones han hecho los agricultores con énfasis en sabor. Algunos de ellos podrían ser usados directamente como variedades comerciales o incorporadas a programas de mejoramiento como fuente de germoplasma para generar variedades o híbridos.

En resumen, el Grupo II mostró los valores más altos en sólidos solubles totales, y el Grupo III sobresalió en rendimiento y tamaño del fruto, así como en firmeza. Según Ramos et al. (2010), esta última característica se considera importante para la comercialización en fresco del tomate. Los materiales experimentales superaron el umbral mínimo para comercialización, que es de $1.45 \mathrm{~N}$ (Batu, 2004). Esto indica que estos materiales tienen potencial para su comercialización local, o que de ellos se puedan generar líneas con buena firmeza para su uso directo por los productores o 


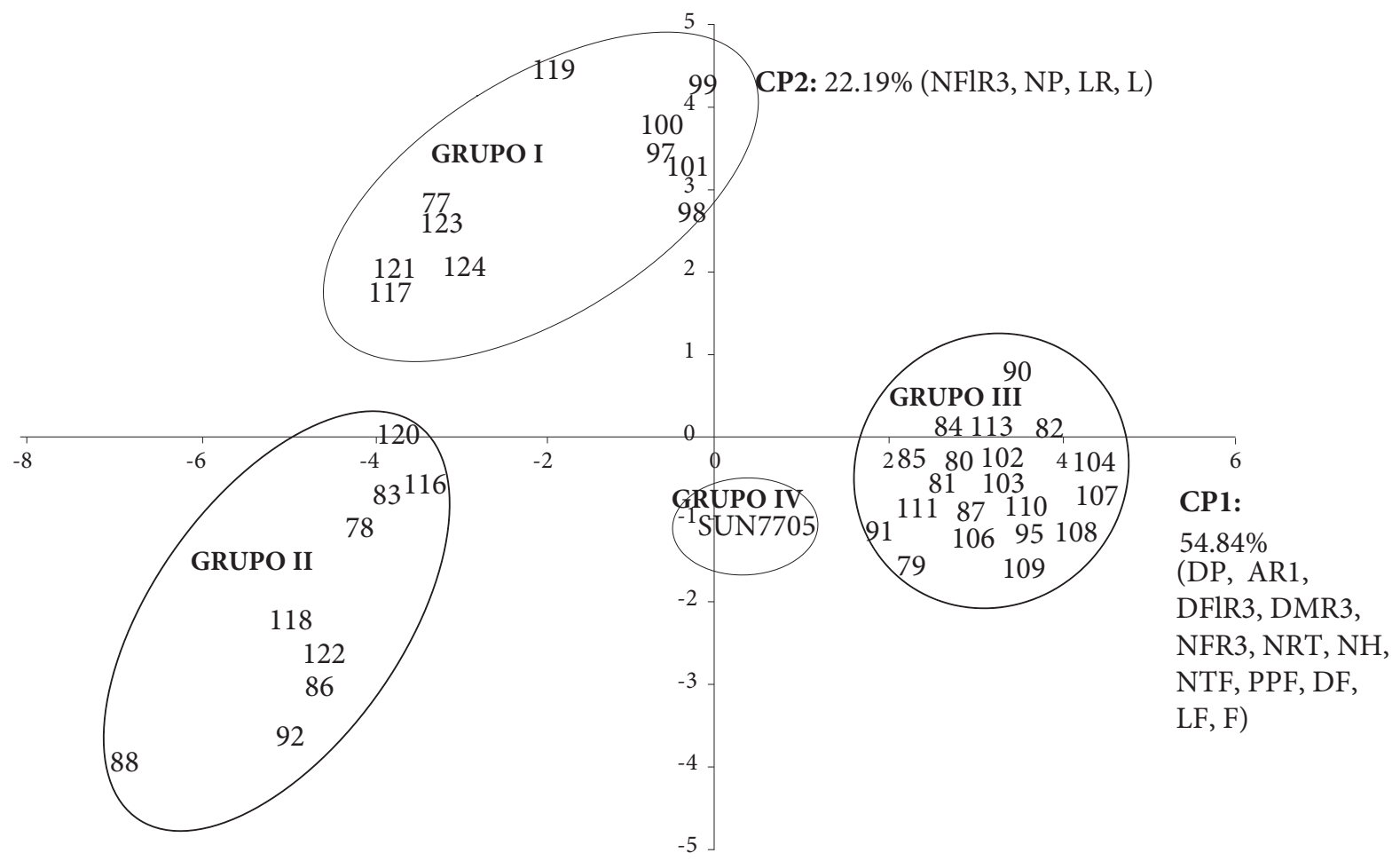

Figura 1. Dispersión de 40 colectas nativas de tomate y un híbrido comercial como testigo, sobre el plano determinado por los primeros dos componentes principales, con base en 20 variables del análisis combinado. DP = diámetro de planta $(\mathrm{mm})$; AR1 = altura al primer racimo $(\mathrm{cm})$; DFIR3 = días a floración del 3er racimo; NFIR3 = número de flores del 3er racimo; DMR3 = días a maduración del fruto del 3er racimo; NFrR3 = número de frutos del 3er racimo; $\mathrm{NRT}$ = número de racimos totales (flor + fruto); $\mathrm{NH}$ = número de hojas; $\mathrm{NP}$ = número de pétalos; $\mathrm{LR}=$ longitud de racimo $(\mathrm{cm}) ; \mathrm{NTF}$ = número total de frutos; PPF = peso promedio de fruto $(\mathrm{g}) ; \mathrm{DF}=$ diámetro de fruto $(\mathrm{mm}) ; \mathrm{LF}=$ longitud de fruto $(\mathrm{mm}) ; \mathrm{F}=$ firmeza $(\mathrm{N}) ; \mathrm{L}=$ número de lóculos.

para mejoramiento.

El contenido de sólidos solubles totales en tomate oscila de 4 a $6{ }^{\circ}$ Brix, fluctuación que depende del riego y otros factores ambientales (Santiago et al., 1998). Las colectas con frutos de tipo "cherry" resultaron dentro del rango propuesto por Santiago et al. (1998), con un valor de $4.44^{\circ}$ Brix que supera al testigo, mientras que en los frutos tipo "riñón" y "saladette" (testigo) los valores fueron de 3.88 y $3.99^{\circ}$ Brix, respectivamente. El aumento en el contenido de sólidos solubles produce también aumentos en el sabor (Nuez, 2001) y en el rendimiento industrial. De hecho, la firmeza y los sólidos solubles totales son considerados como los criterios de calidad más importantes para el fruto de tomate (Jones, 1999; Batu, 2004).

El Grupo IV quedó conformado únicamente por el testigo, el cual fue el híbrido comercial 'SUN7705' que superó a los demás grupos en rendimiento de fruto, con $1570.68 \mathrm{~g}$ por planta, y con un peso promedio del fruto de $104.71 \mathrm{~g}$. El segundo y tercer lugar entre los materiales más rendidores fueron las colectas LOR-107 y LOR-109, con 645 y 702 g por planta, cuyos pesos promedio del fruto fueron de $92 \mathrm{y}$ $80 \mathrm{~g}$, respectivamente.

El análisis de conglomerados formó cuatro grupos y dos subgrupos (Figura 2), los cuales difirieron en su conformación con los obtenidos en el análisis de componentes principales. Esta diferencia se debe a que en el análisis de componentes principales se utilizan únicamente las variables que explican los CP1 y CP2 (77 \% de la variación), mientras que el análisis de conglomerados utiliza todas las variables evaluadas en la obtención del dendrograma.

En el Grupo A se ubicaron las colectas de mayor altura: LOR-77, LOR-83, LOR-116, LOR-117, LOR-120, LOR-121, LOR-122, y LOR-124. Además tuvieron mayor número de flores, de racimos florales, de frutos por racimo, los racimos más largos, y mayor número de racimos totales que el resto de los grupos. Los frutos de estos materiales fueron en su mayoría del tipo "riñón", y sólo dos colectas fueron "ojo de venado", lo que explica su mayor promedio en número 

Cuadro 4. Promedios de la variación de cuatro grupos formados en el análisis de componentes
principales de 40 colectas de tomates nativos y un híbrido comercial como testigo.

\begin{tabular}{lccccc}
\hline VAR & Grupo I & Grupo II & Grupo III & Grupo IV & DMS \\
\hline DP & $13.80 \mathrm{a}$ & $12.46 \mathrm{c}$ & $14.86 \mathrm{a}$ & $13.17 \mathrm{bc}$ & 0.84 \\
A95 & $176.90 \mathrm{ab}$ & $168.03 \mathrm{~b}$ & $141.40 \mathrm{c}$ & $186.18 \mathrm{a}$ & 14.52 \\
AR1 & $30.58 \mathrm{bc}$ & $25.39 \mathrm{c}$ & $43.46 \mathrm{a}$ & $32.46 \mathrm{~b}$ & 5.96 \\
DR & $15.73 \mathrm{a}$ & $11.79 \mathrm{~b}$ & $15.89 \mathrm{a}$ & $16.53 \mathrm{a}$ & 0.99 \\
DFlR3 & $94.32 \mathrm{~b}$ & $87.63 \mathrm{~b}$ & $109.75 \mathrm{a}$ & $88.9 \mathrm{~b}$ & 7.09 \\
NFIR3 & $12.69 \mathrm{a}$ & $8.62 \mathrm{~b}$ & $8.12 \mathrm{~b}$ & $7.72 \mathrm{~b}$ & 1.00 \\
DMR3 & $146.96 \mathrm{~b}$ & $139.67 \mathrm{c}$ & $160.14 \mathrm{a}$ & $143.91 \mathrm{bc}$ & 6.24 \\
NFR3 & $7.04 \mathrm{a}$ & $6.84 \mathrm{a}$ & $6.61 \mathrm{a}$ & $6.01 \mathrm{~b}$ & 0.47 \\
NRT & $7.66 \mathrm{~b}$ & $8.98 \mathrm{a}$ & $5.31 \mathrm{c}$ & $7.63 \mathrm{~b}$ & 0.62 \\
NH & $36.07 \mathrm{~b}$ & $37.62 \mathrm{a}$ & $31.70 \mathrm{c}$ & $32.16 \mathrm{c}$ & 1.33 \\
NP & $7.65 \mathrm{a}$ & $5.43 \mathrm{c}$ & $6.23 \mathrm{~b}$ & $5.67 \mathrm{c}$ & 0.41 \\
LR & $24.17 \mathrm{a}$ & $16.86 \mathrm{~b}$ & $18.89 \mathrm{~b}$ & $18.2 \mathrm{~b}$ & 2.19 \\
NTF & $15.22 \mathrm{c}$ & $18.44 \mathrm{~b}$ & $10.73 \mathrm{~d}$ & $23.58 \mathrm{a}$ & 2.19 \\
PT & $308.22 \mathrm{cb}$ & $143.08 \mathrm{c}$ & $425.40 \mathrm{~b}$ & $1570.68 \mathrm{a}$ & 180.8 \\
PPF & $33.32 \mathrm{c}$ & $12.32 \mathrm{~d}$ & $81.25 \mathrm{~b}$ & $104.71 \mathrm{a}$ & 7.81 \\
DF & $37.44 \mathrm{~b}$ & $23.44 \mathrm{c}$ & $50.43 \mathrm{a}$ & $50.89 \mathrm{a}$ & 2.85 \\
LF & $29.36 \mathrm{c}$ & $21.29 \mathrm{~d}$ & $56.08 \mathrm{~b}$ & $68.84 \mathrm{a}$ & 3.78 \\
F & $0.92 \mathrm{~b}$ & $0.69 \mathrm{~b}$ & $1.48 \mathrm{a}$ & $1.80 \mathrm{a}$ & 0.37 \\
L & $4.89 \mathrm{a}$ & $2.73 \mathrm{bc}$ & $2.93 \mathrm{~b}$ & $2.45 \mathrm{c}$ & 0.30 \\
B & $3.88 \mathrm{~b}$ & $4.44 \mathrm{a}$ & $4.0203 \mathrm{~b}$ & $3.99 \mathrm{~b}$ & 0.37 \\
\hline
\end{tabular}

Medias con letras iguales no son estadísticamente diferentes (Tukey, 0.05); DMS = diferencia mínima significativa; DP = diámetro de planta $(\mathrm{mm}) ;$ A95 = altura de planta a los $95 \mathrm{~d}(\mathrm{~cm}) ; \mathrm{AR} 1=$ altura al primer racimo $(\mathrm{cm}) ; \mathrm{DR}=$ distancia entre racimos $(\mathrm{cm})$; DFlR3 = días a floración del 3er racimo; NFIR3 = número de flores del 3er racimo; DMR3 = días a maduración del fruto del 3er racimo; NFrR3 = número de frutos del 3er racimo; NRT = número de racimos totales (flor + fruto); $\mathrm{NH}$ = número de hojas; $\mathrm{NP}$ = número de pétalos; $\mathrm{LR}=$ longitud de racimo $(\mathrm{cm}) ; \mathrm{NTF}=$ número total de frutos; $\mathrm{PT}$ $=$ peso total de frutos $(\mathrm{g}) ; \mathrm{PPF}=$ peso promedio de fruto $(\mathrm{g}) ; \mathrm{DF}=$ diámetro de fruto $(\mathrm{mm}) ; \mathrm{LF}=$ longitud de fruto $(\mathrm{mm}) ; \mathrm{F}$ = firmeza $(\mathrm{N}) ; \mathrm{L}=$ número de lóculos; $\mathrm{B}=$ sólidos solubles totales $\left({ }^{\circ} \mathrm{Brix}\right)$.

de lóculos.

En el Grupo B incluyó a las colectas con frutos pequeños y precoces: LOR-78, LOR-86, LOR-88, LOR-92, y LOR-118, y por tener los valores más bajos en variables como altura al primer racimo, número de lóculos y firmeza. En sólidos solubles totales tuvieron valores altos.

Los Grupos A y B presentaron características de interés como precocidad y valores altos en sólidos solubles, que son criterios de calidad importantes. Esto hace a estos materiales interesantes para los consumidores y para los investigadores, por la diversidad genética dentro de las colectas factible de ser aprovechada en el mejoramiento genético de la especie.

En el Grupo C se encontraron las colectas LOR-79, LOR-
80, LOR-81, LOR-84, LOR-85, LOR-87, LOR-91, LOR-95, LOR-102, LOR-103, LOR-104, LOR-106, LOR-107, LOR108, LOR-109, LOR-110, LOR-111, y LOR-113. Estas colectas producen frutos del tipo "cuadrado", pero dos fueron arriñonadas (LOR-82 y LOR-90). Este grupo tiene un gran potencial de uso en el corto y mediano plazo porque incluye materiales sobresalientes en tamaño del fruto y en contenido de sólidos solubles, que pueden aprovecharse en el mejoramiento genético de materiales comerciales tipo "saladette" de uso local y nacional.

En el Grupo D se ubicaron las colectas LOR-97, LOR-98, LOR-99, LOR-100, LOR-101, LOR-119, y LOR-123, cuyas inflorescencias tuvieron mayor número de sépalos y frutos con mayor número de lóculos, lo que les confiere la forma arriñonada. Estas colectas también tuvieron los racimos más largos. 


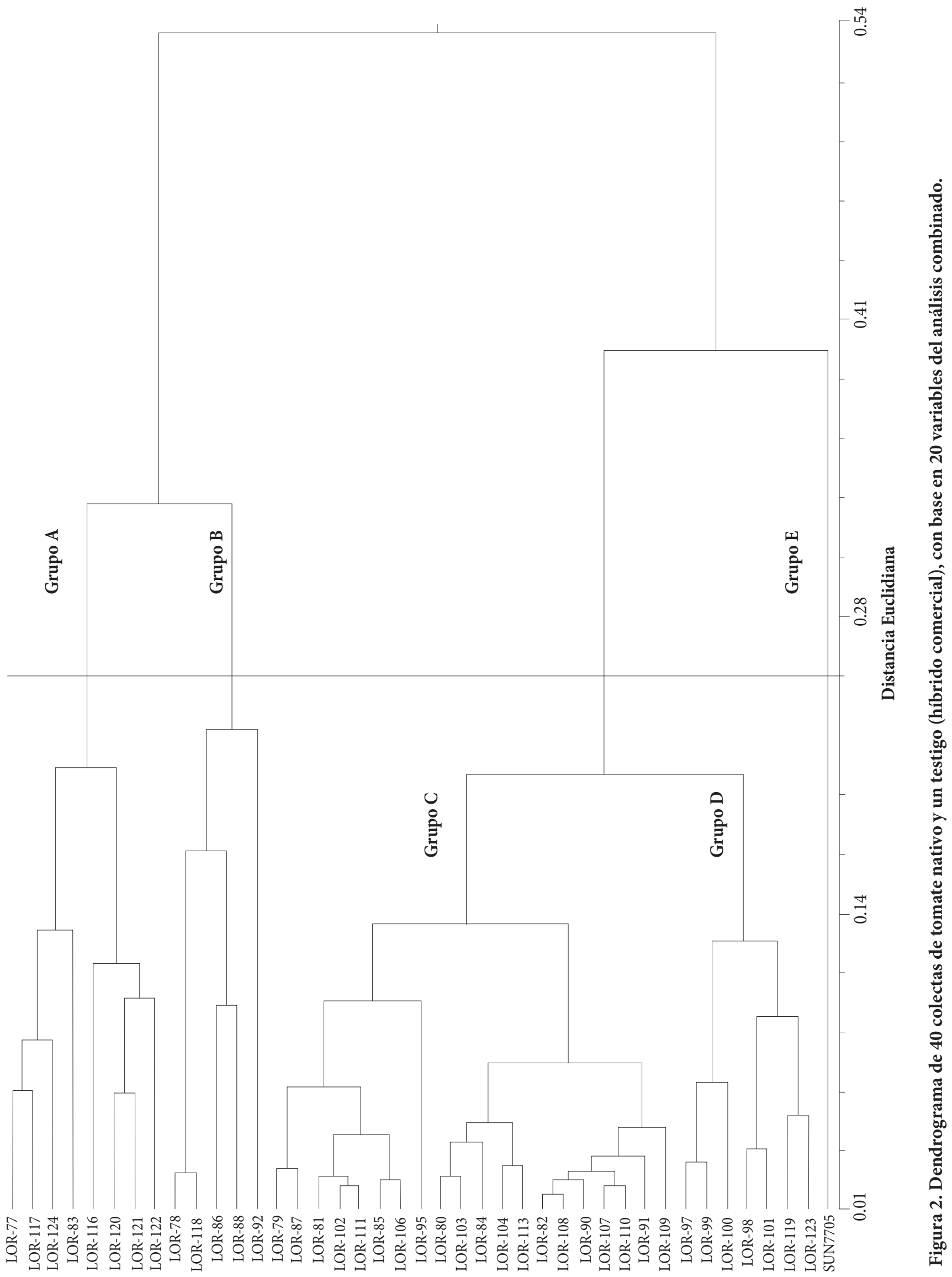


Finalmente, el Grupo E estuvo constituido únicamente por el híbrido testigo el cual sobresalió en la mayoría de las variables. Las características más sobresalientes de este híbrido fueron los componentes de rendimiento y las variables del fruto.

\section{CONCLUSIONES}

Se identificaron colectas con características sobresalientes en sólidos solubles totales y precocidad, como algunas de tipo "cereza", "ojo de venado" y "arriñonados", mismas que podrían utilizarse directamente como variedades de uso local o como fuente de germoplasma.

Los tomates "cuadrados" o tipo "pimiento" sobresalieron en variables de tamaño de fruto, sólidos solubles y firmeza, y presentaron características similares al testigo ('SUN7705'). Tienen un gran potencial para ser usados directamente como variedades o fuente de germoplasma para la generación de variedades e híbridos comerciales tipo "saladette", el cual es de amplio uso a nivel nacional.

Las variables como precocidad, diámetro de planta, componentes del rendimiento y calidad de fruto, fueron las características de mayor importancia que permitieron la formación de grupos, y en las cuales se sugiere poner énfasis en posteriores caracterizaciones de germoplasma nativo mexicano.

\section{BIBLIOGRAFÍA}

Álvarez-Hernández J. C., H. Cortez-Madrigal e I. García-Ruiz (2009) Exploración y caracterización de poblaciones silvestres de jitomate (Solanaceae) en tres regiones de Michoacán, México. Polibotánica 28:139-159.

Bai Y. and P. Lindhout (2007) Domestication and breeding of tomatoes: What have we gained and what can we gain in the future?. Annals of Botany 100:1085-1094.

Batu A. (2004) Determination of acceptable firmness and color values of tomatoes. Journal of Food Engineering 62:472-475.

Bellon M. R. (1996) The dynamics of crop infraspecific diversity: A conceptual framework at the farmer level. Economic Botany 50:2639.

Carrillo-Rodríguez J. C. y J. L. Chávez-Servia (2010) Caracterización agromorfológica de muestras de jitomate de Oaxaca. Revista Fitotecnia Mexicana 33:1-6.

Carrillo-Rodríguez J. C., H. López-Mendoza, J. L. Chávez-Servia, E. Rodríguez-Guzmán, P. Sánchez-Peña and R. Lobato-Ortiz (2012) Phenotypic divergence on growth and productivity of wild and semi-domesticated cherry tomato grown under greenhouse conditions. Acta Horticulturae 947:375-380.

Crisanto-Juárez A. U., A. M. Vera-Guzmán, J. L. Chávez-Servia y J. C. Carrillo-Rodríguez (2010) Calidad de frutos de tomates silvestres (Lycopersicon esculentum var. cerasiforme Dunal) de Oaxaca, México. Revista Fitotecnia Mexicana 33:7-13.

Eigenbrode S. D., J. T. Trumble and R. A. Jones (1993) Resistance to beet armyworm, Hemipterans, and Liriomyza spp. In: Lycopersicon accessions. Journal of the American Society of Horticultural Science 118:525-530.

FAOSTAT, FAO Statistical Database (FAOSTAT) (2008) Area harvested, yield and production 2008 in Mexico. http://faostat.fao.org/ DesktopDefault.aspx? Page ID=339\&lang=es. (Enero 2013).
García-Martínez S., L. Andreani, M. García-Gusano, F. Geuna and J. J. Ruiz (2005) Evaluation of amplified length polymorphism and simple sequence repeats for tomato germoplasm fingerprinting: utility for grouping closely related traditional cultivars. Genome 49:648-656.

Hernández-Leal E., R. Lobato-Ortiz, J. J. García-Zavala, D. ReyesLópez, A. Méndez-López, O. Bonilla-Barrientos y A. Hernández-Bautista (2013) Comportamiento agronómico de poblaciones $\mathrm{F}_{2}$ de híbridos de tomate (Solanum lycopersicum L.). Revista Fitotecnia Mexicana 36:209-215

Hoyt E. (1992) Conservando los Parientes Silvestres de las Plantas Cultivadas. Trad. Enrique Forero. Ed. Addison-Wesley Iberoamericana. Delaware, USA. $52 \mathrm{p}$

IPGRI, Instituto Internacional de Recursos Fitogenéticos (1996) Descriptores de Tomate (Lycopersicon spp.). Ed. Instituto Internacional de Recursos Fitogenéticos, Roma Italia. 49 p.

Jenkins J. A. (1948) The origin of the cultivated tomato. Economic Botany 2:379-392.

Jones J. B. (1999) Tomato Plant Culture. Ed. CRC-Press. Boca Ratón, Florida, USA. $199 \mathrm{p}$

Juárez-López P., R. Castro-Brindis, T. Colinas-León, P. Ramírez-Vallejo, M. Sandoval-Villa, D. W. Reed, L. Cisneros-Zevallos y S. King (2009) Evaluación de calidad de frutos de siete genotipos nativos de jitomate (Lycopersicon esculentum var. cerasiforme). Revista Chapingo. Serie Horticultura 15:5-9.

Lobato-Ortiz R., E. Rodríguez-Guzmán, J. C. Carrillo-Rodríguez, J. L. Chávez-Servía, P. Sánchez-Peña y A. Aguilar-Meléndez (2012) Exploración, Colecta y Conservación de Recursos Genéticos de Jitomate: Avances de la Red de Jitomate. Sistema Nacional de Recursos Fitogenéticos para la Alimentación y Agricultura (SINAREFI). Ed. Secretaría de Agricultura, Ganadería, Desarrollo Rural, Pesca, y Alimentación y Colegio de Postgraduados. Texcoco, México. $54 \mathrm{p}$.

Martínez B. E. (2003) Análisis de acumulación de azucares en pericarpios de dos genotipos nativos de jitomate (Lycopersicon esculentum). Agrociencia 37:363-370.

Miller J. C. and S. D. Tanksley (1990) RFLP analysis of phylogenetic relationships and genetic variation in the genus Lycopersicon. Theoretical and Applied Genetics 80:437-448.

Nieto A. R. y H. E. Velasco (2006) Cultivo de Jitomate en Hidroponia e Invernadero. 2a ed. Ed. Departamento de Fitotecnia, Universidad Autónoma de Chapingo. Chapingo, México. 100 p.

Nuez F. (2001) El Cultivo de Tomate. Ed. Mundi-Prensa. Bilbao, España. $793 \mathrm{p}$.

Park Y. H., M. A. L .West and D. A. St-Clair (2004) Evaluation of AFLPs for germplasm fingerprinting and assessment of genetic diversity in cultivars of tomato (Lycopersicon esculentum L.). Genome 47:510-518.

Peralta I. E. and D. M. Spooner (2007) History, Origin and Early Cultivation of Tomato (Solanaceae). In: Genetic Improvement of Solanaceous Crop, Vol. 2: Tomato. M. K. Razdan and A. K. Mattoo (eds). Science Publishers. Enfield, New Hampshire, USA pp:1-24.

Pérez G. M., F. S. Márquez y A. L. Peña (1997) Mejoramiento Genético de Hortalizas. Ed. Universidad Autónoma Chapingo. Chapingo, México. pp:149-181.

Ramos G. M., S. B. Bautista, N. L. Barrera, M. E. Bosques y C. M. Estrada (2010) Compuestos antimicrobianos adicionados en recubrimientos comestibles para uso en productos hortofrutícolas. Revista Mexicana de Fitopatología 28:44-57.

Rick C. M. (1976) Tomato, Lycopersicon esculentum (Solanaceae). In: Evolution of Crop Plants, N. W. Simmonds (ed). Ed. Longman Group. London. pp:268-273.

Rick C. M. and J. F. Fobes (1975) Allozyme variation in the cultivated tomato and closely related species. Bulletin of the Torrey Botanical Club 102:376-384.

Rick C. M., H. Laterrot and J. Philouze (1990) Andean revised key for the Lycopersicon species. Tomato Genetics Cooperative Report 40:31.

Rohlf F. J. (2000) NTSYS. Numerical Taxonomy and Multivariate Analysis System. Version 2.0, Exeter Publ. Setauket, New York. 31 p.

Sánchez-Peña P., K. Oyama, J. Núñez-Farfán, J. Fornoni, S. HernándezVerdugo, J Márquez-Guzmán and J. A. Garzón-Tiznado 
(2006) Sources of resistance of whitefly (Bemisia spp.) in wild populations of Solanum lycopersicum var. cerasiforme (Dunal) Spooner G J, Anderson et R K Jansen, in Northwestern Mexico. Genetic Resources and Crop Evolution 53:711-719.

Santiago J., M. Mendoza y F. Borrego (1998) Evaluación de tomate ( $L y c o-$ persicum esculentum Mill.) en invernadero: criterios fenológicos y fisiológicos. Agronomía Mesoamericana 9:59-65.
SAS Institute (2002) User's Guide of SAS (Statistical Analysis System). SAS Institute Inc. Cary, N. C. USA. 550 p.

Steiner A. A. (1984) The Universal Nutrient Solution. In: Proceedings 6th International Congress on Soilless Culture. Wageningen, The Netherlands. pp:633-650.

Young T. E., J. A. Juvic and J. G. Sullivan (1993) Acumulation of the components of total solids in ripening fruits of tomato. Journal of the American Society for Horticultural Science 118:286-292. 